\title{
Preferência por local de oviposição de Aedes albopictus (Skuse) (Diptera, Culicidae), em relação à presença de imaturos da própria espécie, sob condições de laboratório ${ }^{1}$
}

\author{
Andreia Aparecida Barbosa 2,3 \\ Mário Antônio Navarro da Silva ${ }^{2}$
}

\begin{abstract}
Aedes (Stegomyia) albopictus (Skuse) (Diptera, Culicidae), preference for oviposition site related with homospecific immatures presence, under laboratory conditions. Aedes (Stegomyia) albopictus (Skuse, 1894) is an exotic Culicidae species in Brazil. Since its first report in this country, the mosquito has been increasing its geographic distribution. This mosquito is a natural dengue and Japanese Encephalitis virus vector in Asia. The females preference for oviposition sites related with homospecific immature presence was assessed. The experiment was performed with Aedes albopictus from laboratory colony since March 1999, in the Laboratório de Entomologia Médica e Veterinária, Departamento de Zoologia, Universidade Federal do Paraná. The preferred container was the one that kept pupae for 24 hours, with 643 eggs, $30,6 \%$ at total. The eggs recipients received $11,45 \%$ from total set by the females, and the following numbers to the others: larva $1(15,79 \%)$, larva 2 $(14,69 \%)$, pupa $1(20,74 \%)$, pupa $2(30,58 \%)$, control $(6,75 \%)$. Although the ANOVA did not detect significant difference among the treatments, the data possibly indicate that Aedes albopictus prefer laying eggs in containers previously colonized by immature.

KEY WORDS. Diptera, Culicidae, Aedes albopictus, oviposition, immature
\end{abstract}

Aedes (Stegomyia) albopictus (Skuse, 1894) é espécie de recente introdução no Brasil e nas Américas. Possui potencialmente condições de exercer o papel de vetor do arbovírus da dengue e febre amarela (FORATTINI 1986; FORATTINI et al. 1997; MARQUES \& GOMES 1997).

No Brasil têm sido notificada sua ocorrência tanto para ecossistemas silvestres quanto para grandes centros urbanos (SANT'ANA 1996; NATAL et al. 1997), indicando a capacidade de tal culicídeo explorar um gradiente de ambientes. Para GOMES et al. (1992) a cepa de Aedes albopictus (Skuse, 1894) presente no Vale do Paraíba (Estado de São Paulo, Brasil), parece ser oriunda da Ásia tropical.

Em território brasileiro sua presença foi assinalada em uma série de criadouros, tanto naturais quanto artificiais. Dentre os naturais, citam-se as bromélias, ocos de árvore, cascas de frutas, internódios de bambu, entre outros (NATAL et al. 1997; GOMES \& MARQUES 1988; SANTOS \& NASCIMENTO 1998). Associado à presença humana, pode colonizar recipientes produzidos por sua interferência. Os criadouros

1) Contribuição número 1386 do Departamento de Zoologia, Universidade Federal do Paraná.

2) Departamento de Zoologia, Universidade Federal do Paraná. Caixa Postal 19020, 81531-990 Curitiba, Paraná, Brasil. E-mail: mnavarro@bio.ufpr.br

3) Bolsista CNPq. E-mail: deiaguel@bio.ufpr.br

Revta bras. Zool. 19 (4): 1147 - 1152, 2002 
artificiais incluem uma série de recipientes, muitos deles produzidos pela ação antrópica. Citam-se nesta categoria alguns exemplos como: pneus, sucatas de veículos e eletrodomésticos, entulhos de construção civil e recipientes plásticos. (FORATTINI et al. 1998; CALADO \& NAVARRO-Silva 2002).

A escolha do local de oviposição pelas fêmeas grávidas é provavelmente influenciada por uma série de fatores endógenos e exógenos, determinando o sucesso reprodutivo da espécie e possibilitando que o ciclo evolutivo seja completado (HWANG et al. 1980; BENTLEY \& DAY 1988; BENZON \&APPERSON 1988; ZAHIRI \& RAU 1998).

As duas espécies exóticas de Aedes Meigen, 1818 que utilizam o ecossistema urbano no Brasil, Aedes aegypti Linnaeus, 1762 e Aedes albopictus, possuem estratégias semelhantes de oviposição, distribuindo seus ovos de forma parcelada nos criadouros, potencializando as condições de manutenção da população neste ecossistema (ROZEBOOM et al. 1973).

O conhecimento sobre o comportamento de oviposição de Aedes albopictus pode levar ao desenvolvimento de criadouros iscas, utilizando o extrato de imaturos mais atrativo para as fêmeas grávidas e também para o monitoramento precoce da presença desta espécie nos ecossistemas antrópicos.

O objetivo deste trabalho foi a busca de indícios de preferência por local de oviposição para Aedes albopictus.

\section{MATERIAL E MÉTODOS}

A colônia de Aedes albopictus utilizada para a realização dos experimentos era proveniente da cidade de Registro (Vale do Ribeira, Estado de São Paulo) a partir de imaturos obtidos em área urbana. Tais exemplares foram coletados em março de 1999 e mantidos no Laboratório de Entomologia Médica e Veterinária do Departamento de Zoologia da Universidade Federal do Paraná.

Isolou-se em uma gaiola de criação, com dimensões de $50 \times 50 \mathrm{~cm}$, um lote de 100 indivíduos recém emergidos, sendo 50 fêmeas e 50 machos, que receberam diariamente alimentação sangüínea e solução de mel a 10\%. A gaiola teste foi mantida em sala climatizada sob variação natural de luz, temperatura e umidade relativa do ar oscilando respectivamente entre 18 e $27^{\circ} \mathrm{C}$ e 74 e $82 \%$. Estes dois últimos parâmetros ambientais foram monitorados através de termo-higrômetro digital.

Transcorridos três dias, iniciou-se o experimento de local de preferência das fêmeas de Aedes albopictus porem seus ovos, introduzindo-se na gaiola teste seis potes plásticos com capacidade para $120 \mathrm{ml}$ de água e mesma coloração, com os seguintes tratamentos: a) Controle: apenas água submetida a aeração, b) Ovos: água com 50 ovos, c) Larva 1: água com 20 larvas de quarto instar, d) Larva 2: água na qual permaneceram larvas de quarto instar por 24 horas, e) Pupa 1: água com 20 pupas com menos de 24 horas de formadas e f) Pupa 2: água na qual permaneceram pupas por 24 horas.

Para cada conjunto de seis tratamentos, foram realizadas dez réplicas. Diariamente, os potes foram retirados da gaiola teste, sendo verificada a presença de ovos e anotado o número dos mesmos. Após este procedimento, novos conjuntos 
de tratamentos foram introduzidos na gaiola teste, obedecendo à posição previamente estabelecida e realizando em cada réplica o giro dos recipientes no sentido horário, para evitar o efeito de localização dos tratamentos.

A preferência em relação à variável oviposição foi testada através da análise de variância de um fator, tendo sido os dados previamente transformados em log $(\mathrm{x}+1)$, necessário par atingir a homogeneidade dos dados.

\section{RESULTADOS E DISCUSSÃO}

Durante a realização dos experimentos, as fêmeas de Aedes albopictus ovipositaram um total de 2103 ovos em recipientes com diferentes estágios e tratamentos das formas evolutivas da própria espécie.

A escolha do local para a oviposição pode ser influenciada por vários fatores físicos, químicos e biolọgicos e o local escolhido para deposição dos ovos pode determinar diferenças na taxa de mortalidade e média do comprimento das asas das fêmeas (GOMES et al. 1995).

A presença de formas evolutivas de Aedes albopictus pode atuar como um fator atrativo importante para as fêmeas, pois podem indicar que o criadouro seja viável para reprodução da espécie possuindo alimento, condições ideais de oxigenação, temperatura e faixa de $\mathrm{pH}$ adequados. A escolha dos locais de oviposição apresenta-se como fator crítico para sobrevivência e dinâmica das populações. Possui importantes implicações no controle de mosquitos, sendo a escolha de tais locais, resultado de uma complexa interação entre fatores químicos e físicos, mostrando também alto grau de preferência na seleção dos locais de oviposição, HWANG et al. (1980), BENZON \& APPERSON (1988) e BENTLEY \& DAY (1988).

Comparando-se os recipientes com a presença de imaturos e o controle, ocorreu postura de ovos superior em aproximadamente duas a quatro vezes ao controle, indicando que a presença de imaturos da própria espécie, independente do estágio de desenvolvimento atuou de forma positiva na preferência das fêmeas em depositarem seus ovos. A proporção de ovos depositados no experimento aumentou de acordo com o avanço do estágio evolutivo. Considerando apenas os resultados percentuais, pode-se indicar que as fêmeas de Aedes albopictus apresentam tendência em realizar oviposição em recipientes com imaturos da própria espécie, visto que este fator pode indicar para as fêmeas grávidas a viabilidade ou não do criadouro.

Apesar da diferença observada entre as porcentagens de ovos depositados nos recipientes, não foi detectada diferença significativa entre os recipientes oferecidos para oviposição das fêmeas ( $\left.\mathrm{F}_{5}, 54=1,5, \mathrm{p}>0,05\right)$ (Fig. 1).

As oviposições de fêmeas de Aedes albopictus aumentaram significativamente em resposta a água de criação da própria espécie, quando comparadas aos recipientes controle, porém não sendo afetada a resposta de oviposição pela presença de ovos de outras espécies (ALLAN \& KLINE 1998). Consideração similar foi apresentada por YAP et al. (1995) onde água ocupada previamente por forma imaturas de Aedes albopictus exerce atração sobre fêmeas grávidas desta espécie.

MARQUES \& MIRANDA (1992) testaram a influência de extratos de formas evolutivas sobre a atividade de fêmeas de Aedes albopictus. Extratos aquosos de larvas, pupas e ovos foram testados, sendo que os de larvas e pupas tiveram um 


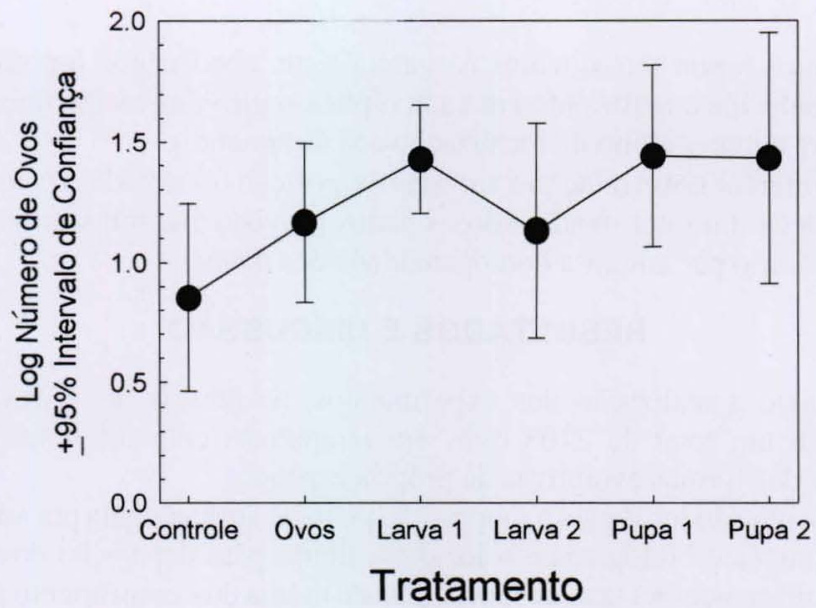

Fig. 1. Logaritmo do número de ovos depositados nos recipientes controle (ága submetida a aeração), ovos (água com 50 ovos), Larva 1 (água com 20 larvas de quarto instar), Larva 2 (água na qual permaneceram larvas de quarto instar por 24 horas), Pupa 1 (água com 20 pupas com menos de 24 horas de formadas) e Pupa 2 (água na qual permaneceram pupas por 24 horas).

efeito atrativo sobre as fêmeas e o de ovos não exerceram influência sobre o comportamento de oviposição. Em nossos resultados, embora com a proporção mais reduzida, os recipientes com ovos receberam $11,45 \%$ do total depositados, com os seguintes percentuais para demais recipientes: Larva 1 (15,79\%), Larva $2(14,69 \%)$, Pupa $1(20,74 \%)$ e Pupa $2(30,58 \%)$, com os recipientes com larvas e pupas exercendo atração superior aos ovos. No recipiente utilizado como controle, foram depositados $6,75 \%$ do total de ovos.

A resposta de oviposição de fêmeas grávidas de Aedes albopictus foi testada por ALLAN \& KLINE (1995), frente à infusões orgânicas (infusão de feno, água de criação de larvas e água coletada em criadouros naturais de larvas) e os compostos sintéticos isolados da infusão de feno (3-metilindol, 4-metilfenol, indol e fenol). Em laboratório, foram obtidas respostas significativas para a oviposição de Aedes albopictus em relação a diluições do infuso de feno e água do criadouro natural. Os compostos sintéticos causaram respostas moderadas de oviposição. Nas avaliações feitas em campo, a resposta de oviposição de Aedes albopictus foi muito maior para a água de criação das larvas e para a água do criadouro natural, moderadas para a infusão de feno e 3-metilindol.

TREXLER et al. (1998), para Aedes albopictus, realizaram experimentos em laboratório com infusão de folhas de Quercus alba Linnaeus. Esta infusão agiu como atrativo para oviposição. O maior número de ovos ocorreu em reposta à infusão de folhas em $60 \%$ de concentração. Aedes albopictus, em comparação por preferência de local de oviposição em recipiente contendo água pura ou recipiente contendo infusão, preferiu colocar seus ovos no recipiente contendo infusão, não importando o tempo de permanência das folhas no meio. 
No entanto, levando-se em consideração apenas o resultado da análise de variância, Aedes albopictus poderia demonstrar a não preferência por um sítio particular para a oviposição e a ausência de um efeito causado pela utilização prévia de um criadouro. Estes podem ser considerados fatores epidemiológicos muito importantes. A falta de discriminação pode demonstrar a plasticidade do comportamento de oviposição, constituindo-se em uma característica altamente relacionada com o poder de colonização, que é o processo chave para o estabelecimento, manutenção e dispersão das populações.

AGRADECIMENTOS. Ao professor PhD. James J. Roper, Departamento de Botânica, Universidade Federal do Paraná, pela análise estatística.

\section{REFERÊNCIAS BIBLIOGRAFICAS}

ALLAN, S.A. \& D.L. KLINE. 1995. Evalution of organic and synthetic compounds meadiating oviposition in Aedes albopictus and Aedes aegypti (Diptera: Culicidae). Jour. Chem. Ecol. 21 (11): 1847-1860.

- 1998. Larval rearing water and preexisting eggs influence oviposition by Aedes aegypti and Ae. albopictus (Diptera: Culicidae). Jour. Med. Entomol. 35 (6): 943-947.

BentLEY, M.D. \& J.F. DAY. 1988. Chemical ecology and Behavioral aspects of mosquito oviposition. Annu. Rev. Ent. 34: 401-421.

BENZON, G.L.; \& C.S. APPERSON. 1988. Reexamination of chemically mediated oviposition behavior in Aedes aegypti (L.) (Diptera-Culicidae). Jour. Med. Entomol. 25 (3): 158-182

Calado, D.C. \& M.A. Navarro-Silva. 2002. Influência da temperatura sobre a longevidade, fecundidade e atividade hematofágica de Aedes (Stegomyia) albopictus Skuse, 1894 (Diptera, Culicidae) sob condições de laboratório. Revta bras. Ent. 46 (1): 93-98.

Forattini, O.P. 1986. Identificação de Aedes (Stegomyia) albopictus (Skuse) no Brasil. Rev. Saúde Públ., São Paulo, 20 (3): 244-245.

Forattini, O.P.; I. Kakitani; M.A.M. Sallum \& L. DE Rezende. 1997. Produtividade de criadouro de Aedes albopictus em ambiente urbano. Rev. Saúde Públ., São Paulo, 31 (6): 545-555.

Forattini, O.P.; G.R.A.M. Marques; I. Kakitani; M. DE Brito \& M.A.M. SAlluM. 1998. Significado epidemiológico dos criadouros de Aedes albopictus em bromélias. Rev. Saúde Públ., São Paulo, 32 (2): 523-530.

Gomes, A.C. \& G.R.A.M. MARques. 1988. Encontro de criadouros natural de Aedes (Stegomyia) albopictus (Skuse), no Estado de São Paulo, Brasil. Rev. Saúde Públ., São Paulo, 22 (3): 245.

Gomes, A.C.; O.P. Forattini; I. Kakitani; G.R. Marques; C.C. Marques, D. MarucCI \& M. DE Brito. 1992. Microhabitats de Aedes albopictus (Skuse) na região do Vale do Paraíba, Estado de São Paulo, Brasil. Rev. Saúde Públ., São Paulo, 26 (2): 108-118.

Gomes, A.C.; S.L.D. Gotlieb; C.C.A. Marques, M.B. De Paula \& G.R.A.M. Marques. 1995. Duration of larval and pupal development stages of Aedes albopictus in natural and artificial containers. Rev. Saúde Públ., São Paulo, 29 (1): 15-19.

Hwang, Yhi-Shen, W.L. Kramer \& M.S. Mulla. 1980. Oviposition attractaans and repellents of mosquitoes. J.our Chem. Ecol. 6 (1): 71-80.

MARques, C.C.A. \& C. MIRANDA. 1992. Influência de extratos de formas evolutivas sobre atividades de oviposição de fêmeas de Aedes (S.) albopictus (Skuse). Rev. Saúde Públ., São Paulo, 26 (4): 269-271.

MARques, G.R.A.M. \& A. DE GoMES. 1997. Comportamento antropofílico de Aedes albopictus (Skuse) (Diptera: Culicidae) na região do Vale do Paraíba, Sudeste do Brasil. Rev. Saúde Públ., São Paulo, 31 (2): $125-130$.

Natal, D.; P.R. Urbinatti; C.B. Taipe-Lagos; W. Cereti-Júnior; A.T.B. Diederichsen; R.P. Souza. 1997. Encontro de Aedes (Stegomyia) albopictus (Skuse) em Bromeliaceae na periferia de São 
Paulo, SP, Brasil. Rev. Saúde Públ., São Paulo, 31 (5): 517-518

Rozeboom, L.E; L. Rosen \& J. IKEDA. 1973. Observations on oviposition by Aedes (S.) albopictus Skuse and A.(S.) polynesiensis Marks in nature. Jour. Med. Entomol. 10 (4): 397-339.

SANT'ANA, A.L. 1996. Primeiro encontro de Aedes albopictus (Skuse) no Estado do Paraná, Brasil. Rev. Saúde Públ., São Paulo, 30 (4): 392-393.

Santos, S.O. Dos \& J.C. Do Nascimento. 1998. Primeiro registro da presença do Aedes (Stegomyia) albopictus (Skuse) em Mato Grosso do Sul, Brasil. Rev. Saúde Públ., São Paulo, 32 (5): 486.

TREXLER, J.D.; C.S. ApPERSON \& C. SCHAL. 1998. Laboratory and field evaluations of oviposition responses of Aedes albopictus and Aedes triseriatus (Diptera: Culicidae) to oak leaf infusions. Jour. Med. Entomol. 35 (6): 967-976.

YAP, H.H.; C.Y. LeE; N.L. ChONG; A.E. Foo \& M.P. LiM. 1995. Oviposition site preference of Aedes albopictus in the laboratory. Jour. Amer. Mosq. Control Assoc. 11 (1): 128-132.

ZAHIRI, N. \& M.E. RAU. 1998. Oviposition responses of Aedes aegypti. Jour. Med. Entomol. 35 (5): $782-787$.

Recebido em 24.VI.2002; aceito em 28.X.2002. 